\title{
Psammaplin A-Modified Novel Radiosensitizers for Human Lung Cancer and Glioblastoma Cells
}

\author{
Chan Woo Wee', Jin Ho Kim ${ }^{1,2,3}$, Hak Jae Kim ${ }^{1,2,3}$, Hyun-Cheol Kang' ${ }^{1}$, Soo Youn Suh², Beom Soo Shin ${ }^{4}$, Eunsook Ma ${ }^{5, *}$, \\ II Han Kim ${ }^{1,2,3, *}$ \\ ${ }^{1}$ Department of Radiation Oncology, Seoul National University College of Medicine, Seoul, Korea; ${ }^{2}$ Cancer Research Institute, Seoul National University \\ College of Medicine, Seoul, Korea; ${ }^{3}$ Institute of Radiation Medicine, Seoul National University College of Medicine, Seoul, Korea; ${ }^{4}$ School of Pharmacy, \\ Sungkyunkwan University, Suwon, Korea; ${ }^{5}$ College of Pharmacy, Daegu Catholic University, Gyeongsan, Korea
}

\section{Original Research}

Received November 30, 2018

Revision January 18, 2019

Accepted February 11, 2019

Corresponding author: II Han Kim

Department of Radiation Oncology, Seoul National University College of Medicine, 101 Daehak-ro, Jongno-gu, Seoul 03080, Korea

Tel: $+82-2-2072-2528$

Fax: +82-2-765-3317

E-mail: ihkim@snu.ac.kr

Co-corresponding author: Eunsook Ma

College of Pharmacy, Daegu Catholic University, 13-13 Hayang-ro, Hayang-eup, Gyeongsan 38430, Korea

Tel: +82-53-850-3621

Fax: +82-53-850-3602

E-mail:masook@cu.ac.kr

Chan Woo Wee and Jin Ho Kim have equally contributed to this article.

This is an Open-Access article distributed under the terms of the Creative Commons Attribution NonCommercial License (http://creativecommons.org/ licenses/by-nc/4.0) which permits unrestricted noncommercial use, distribution, and reproduction in any medium, provided the original work is properly cited.

Copyright $\odot$ 2019The Korean Association for Radiation Protection

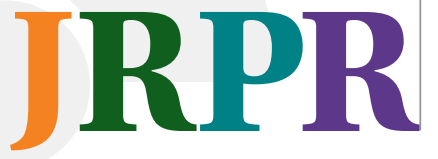

Background: Psammaplin A (PsA) is a radiosensitizer whereas its clinical application is hampered by poor bioavailability. This study aimed to synthesize novel radiosensitizers using PsA as the lead compound.

Materials and Methods: Eight homodimeric disulfides were synthesized from corresponding acid and cystamine dihydrochloride in $\mathrm{N}$-hydroxysuccinimide and dicyclohexylcarbodiimide coupling conditions. One monomeric thiol analog was obtained by reduction of homodimeric disulfide with dithiothreitol. Clonogenic assay was used to measure cell survival after irradiation and drug treatment in human lung cancer (A549) and glioblastoma (U373MG) cells.

Results and Discussion: Using the PsA backbone, nine compounds were synthesized. Eight compounds showed variable cytotoxicity with $50 \%$ inhibitory concentrations ranging $16.14 \mu \mathrm{M}$ to $150.10 \mu \mathrm{M}$ (A549), and $13.25 \mu \mathrm{M}$ to $50.15 \mu \mathrm{M}$ (U373MG). Four and six compounds radiosensitized A549 and U373MG cells, respectively. Two compounds that radiosensitized both cell lines were tested for its inhibitory effects on DNMT1. One of them was shown to significantly inhibit DNMT1 activity.

Conclusion: Novel compounds with radiosensitizing activity were synthesized. These compounds have a great potential to serve as a basis for the development of future radiosensitizers. Further investigation is warranted for their clinical application.

Keywords: Psammaplin A, DNA methyltransferase, Radiosensitizer, Lung cancer, Glioblastoma

\section{Introduction}

Radiotherapy (RT) is highly effective for cancer treatment, with over $50 \%$ of all cancer patients receiving this treatment. The combination of chemotherapeutic agents with RT has significantly improved local control of cancers and patient survival. But some cancers exhibit inherent radiation resistance. The goal of RT is maximizing cell-killing in the cancer cells while minimizing the damage of normal tissues. Studies to improve the therapeutic ratio have resulted in the development of certain compounds that can act to increase the radiosensitivity of cancer cells or to protect the normal cells from RT [1]. Above all, efforts have been made to develop radiosensitizers in order to combine those agents and eventually enhance the efficacy of RT. Not only can radiosensitizers enhance cell lethality at the same RT-doses, it enables to deescalate RT-doses main- 
taining the cell-killing effects which can subsequently result in reduction of radiation exposure of normal tissues is patients.

DNA methylation is mediated by DNA methyltransferase (DNMT), and it plays an important role in epigenetic regulation of genes leading to suppressed gene expression. Aberrant silencing of tumor suppressor genes is known to be associated with development and progression of cancer $[2,3]$. Given that DNMT overexpression has been found in various types of cancers, DNMT inhibitors (DNMTIs) have been investigated as anti-cancer agents [4-6]. DNMTIs are classified into two families, which are nucleosides and non-nucleosides, and various DNMTIs showed anti-cancer activity by themselves or in combination with other cytotoxic agents [710]. As a result, drugs such as 5 -azacytidine (Azacitidine, Vidaza $^{\circledR}$ ) and 5-aza-2'-deoxycytidine (Decitabine, Dacogen $^{\circledR}$ ) have actually been approved by the FDA for treatment of myelodysplastic syndrome as well as acute myeloid leukemia [8]. Nucleoside analogues such as 5-azacytidine, zebularine and 5-aza-2'-deoxycytidine enhanced RT lethality in human cancer cell lines [11-14]. Furthermore, DNMTIs are also known to sensitize cancer cells to cell killing by RT. These results suggest the potential application of DNMTIs as radiosensitizers. However, application of nucleoside DNMTIs in cancer treatment has occasionally been restricted due to their toxicity and poor chemical stability $[15,16]$. Given the limitations of nucleoside analogues, development of non-nucleoside DNMTIs is needed.

Psammaplin A (PsA) is a symmetrical bromotyrosine-derived disulfide dimer isolated from the marine sponge Pseudoceratina purpurea, and it represents the first example of a disulfide and oxime containing natural product [17]. PsA has been found to have a wide range of bioactivities, especially for antimicrobial and antitumor activity [18]. More to the point, PsA showed an inhibitory activity against both DNMT and histone deacetylase [18-20]. We previously reported that PsA, as a non-nucleoside DNMTI, sensitizes human lung cancer and glioblastoma cells to radiation lethality in vitro [21]. However, PsA is unstable in vivo and has poor bioavailability [22]. To extend or maintain the radiosensitizing activity of PsA towards further studies, we aimed to develop novel PsA-modified derivatives that can serve as future backbones for radiosensitizers. In this study we synthesized and screened the PsA-modified compounds for their radiosensitizing efficacy in human lung cancer and glioblastoma cells.

\section{Materials and Methods}

\section{Synthesis}

Moisture- or air-sensitive reaction was conducted under nitrogen in distilled solvents. The standard reaction work-up involved drying the solution of crude product over anhydrous $\mathrm{MgSO}_{4}$, removing the solvent under reduced pressure. The commercial reagents were purchased from Aldrich, Fluka or Sigma Co. Melting points were measured on ThomasHoover melting point apparatus and not corrected. ${ }^{1} \mathrm{H},{ }^{13} \mathrm{C}$, HSQC and HMQC NMR spectra were taken on Varian 400 $\mathrm{MHz}$ spectrometer in $\mathrm{CDCl}_{3}, \mathrm{DMSO}-d_{6}$, or $\mathrm{CD}_{3} \mathrm{OD}$. Chemical shifts $(\delta)$ are in parts per million (ppm) relative to tetramethylsilane, and coupling constants $(J)$ are in Hertz $(\mathrm{Hz})$. The abbreviations in the NMR spectra are as follows; s: singlet, br: broad, d: doublet, dd: doublet of doublet, t: triplet, quint: quintet, m: multiplet, mp: melting point, ArH: aromatic hydrogen. GC-MS (EI) spectra were obtained on a GCMS QP2010 (Shimadzu Corp., Kyoto, Japan) and Agilent 5975C MSD spectrometer (Agilent, Santa Clara, USA). High resolution fast-atom bombardment mass spectra (HRMS [FAB]) were obtained on a JMS-700 spectrometer (Jeol, Peabody, USA). Medium pressure liquid chromatography (MPLC) was performed on YAMAZEN and fraction collector performed on EYELA DC-1500 (Tokyo Rikakikai Co., Ltd., Tokyo, Japan). An analytical thin layer chromatography (TLC) was performed on pre-coated silica gel $60 \mathrm{~F}_{254}$ plates (Merck \& Co., Inc., Kenilworth, USA). Solvent systems for TLC were ethyl acetate $/ n$-hexane and $10 \%$ methanol in dichloromethane. Column chromatography was carried out on Merck silica gel 9385 (230-400 mesh) (Merck \& Co., Inc.) and eluted with ethyl acetate/ $n$-hexane and methanol/dichloromethane mixture.

1) General procedures for the synthesis of compounds 1-3

To a solution of 4-hydroxyphenylpyruvic acid (900 mg, 5.0 $\mathrm{mmol})$ in absolute ethanol $(20 \mathrm{~mL})$, hydroxylamine or $O$ benzylhydoxylamine hydrochloride $(10.0 \mathrm{mmol})$ and trimethylamine $(10.0 \mathrm{mmol})$ were added and stirred for 5 hours at room temperature. The reaction mixture was rotary evaporated to remove ethanol under reduced pressure. The residue was added with water and acidified with $\mathrm{c}-\mathrm{HCl}$ and then extracted with ethyl acetate $(3.30 \mathrm{~mL})$. The combined organic extract was dried with anhydrous $\mathrm{MgSO}_{4}$, filtrated and concentrated to give crude precipitate which was recrystallized with ethyl acetate and $n$-hexane mixture to give pure 
white or pale yellow compound.

(1) 2-(Hydroxyimino)-3-(4-hydroxyphenyl) propanoic acid (1)

Yield: $85 \%$, mp: $156-158^{\circ} \mathrm{C}$ (lit. $153-155^{\circ} \mathrm{C}$ [23])

(2) 2-(Benzyloxyimino)-3-(4-hydroxyphenyl) propanoic acid (2)

Yield: 88\%, mp: 100-102 ${ }^{\circ} \mathrm{C},{ }^{1} \mathrm{H}$ NMR (DMSO- $\left.d_{6}\right)$ : $9.29(1 \mathrm{H}$, br s, $\mathrm{COOH}), 7.28-7.42(5 \mathrm{H}, \mathrm{m}, \mathrm{ArH}), 6.95(2 \mathrm{H}, \mathrm{d}, J=8.8 \mathrm{~Hz}$, H-2,6), 6.64 (2H, d, J=8.4 Hz, H-3,5), 5.25 (2H, s, $\left.\mathrm{CH}_{2}\right), 3.71$ $\left(2 \mathrm{H}, \mathrm{s}, \mathrm{CH}_{2}\right) ;{ }^{13} \mathrm{C}$ NMR (DMSO- $\left.d_{6}\right): 165.3(\mathrm{C}=\mathrm{O}), 154.2$ (C= N), 153.1 (C-4), 135.1, 131.8 (C-2,6), 128.6 (C-1), 128.4, 128.1, $116.3(\mathrm{C}-3,5), 76.3\left(\mathrm{CH}_{2}\right), 37.6\left(\mathrm{CH}_{2}\right)$, GC-MS (EI) $m / z$ : $285[\mathrm{M}]^{+}$.

(3) 2-(Benzyloxyimino)-3-(3-bromo-4-hydroxyphenyl)

propanoic acid (3)

Yield: $78 \%, \mathrm{mp}: 149-151^{\circ} \mathrm{C},{ }^{1} \mathrm{H} \mathrm{NMR}\left(\mathrm{CDCl}_{3}\right)$ : $10.12(1 \mathrm{H}, \mathrm{br} \mathrm{s}$, $\mathrm{COOH}), 7.30-7.37$ (5H, m, ArH), 7.26 (1H, d, J=2.0 Hz, H-2), $6.97(1 \mathrm{H}, \mathrm{dd}, J=8.0,2.0 \mathrm{~Hz}, \mathrm{H}-6), 6.83(1 \mathrm{H}, \mathrm{d}, J=8.0 \mathrm{~Hz}, \mathrm{H}-5)$, 5.26 (2H, s, $\mathrm{CH}_{2}$ ), $3.71\left(2 \mathrm{H}, \mathrm{s}, \mathrm{CH}_{2}\right),{ }^{13} \mathrm{C}$ NMR (DMSO- $d_{6}$ ): 165.9 $(\mathrm{C}=\mathrm{O}), 154.8(\mathrm{C}=\mathrm{N}), 151.3(\mathrm{C}-4), 136.3,132.3,130.0,129.5$, 128.4, 127.8 (C-1), 127.0, 115.6 (C-5), 110.1 (C-3), $76.5\left(\mathrm{CH}_{2}\right)$, $38.8\left(\mathrm{CH}_{2}\right), \mathrm{GC}-\mathrm{MS}(\mathrm{EI}) m / z: 365[\mathrm{M}+1]^{+}, 363[\mathrm{M}-1]^{+}$.

2) General procedures for the synthesis of homodimers (4-6 [24], 8, 10, 12)

To a solution of carboxylic acid (1-3, 3,4-difluorocinnamic acid, cinnamic acid, 2-chlorophenylacetic acid, 3-methoxyphenylacetic acid, and benzoic acid, $1.0 \mathrm{mmol}$ ) in 1,4-dioxane $(20 \mathrm{~mL})$ and dimethylformamide (DMF, $3 \mathrm{~mL}), N$-hydroxysuccinimide (NHS, $1.5 \mathrm{mmol}$ ) and dicyclohexylcarbodiimide (DCC, $1.5 \mathrm{mmol}$ ) were added at room temperature. After stirring for 1 hour, a mixture of cystamine dihydrochloride $(0.5 \mathrm{mmol})$ and triethylamine $(2.0 \mathrm{mmol})$ in 1,4-dioxane $(5 \mathrm{~mL})$ was added at $0^{\circ} \mathrm{C}$ and stirred for 24 hours at room temperature. After the reaction was over, the reaction mixture was left standing in refrigerator and then filtered. The obtained filtrate was washed with brine and extracted with ethyl acetate $(3.30 \mathrm{~mL})$. The extract was dried over anhydrous $\mathrm{MgSO}_{4}$, filtered and concentrated to give a crude compound which was purified by column chromatography (ethyl acetate: $n$-hexane $=1: 1$ and 2:1) or MPLC to give a pure white or pale yellow compound.
(1) N,N'-Bis(3-(3-bromo-4-hydroxyphenyl)-2-

benzyloximinopropionyl)cystamine (4)

Yield: $41 \%$, ${ }^{1} \mathrm{H}$ NMR $\left(\mathrm{CDCl}_{3}\right)$ : 7.32-7.39 (10H, m, ArH), 7.24 ( $\left.2 \mathrm{H}, \mathrm{d}, J=2.0 \mathrm{~Hz}, \mathrm{H}-2,2^{\prime}\right), 7.01\left(2 \mathrm{H}, \mathrm{t}, J=6.0 \mathrm{~Hz}, \mathrm{H}-6,6^{\prime}\right), 6.85$ $\left(2 \mathrm{H}, \mathrm{d}, J=8.0 \mathrm{~Hz}, \mathrm{H}-5,5^{\prime}\right), 5.25\left(4 \mathrm{H}, \mathrm{s}, 2 \cdot \mathrm{CH}_{2}\right), 3.73\left(4 \mathrm{H}, \mathrm{s}, 2 \cdot \mathrm{CH}_{2}\right)$, $3.41\left(4 \mathrm{H}, \mathrm{t}, J=6.8 \mathrm{~Hz}, 2 \cdot \mathrm{CH}_{2}\right), 2.80\left(4 \mathrm{H}, \mathrm{t}, J=6.8 \mathrm{~Hz}, 2 \cdot \mathrm{CH}_{2}\right)$, ${ }^{13} \mathrm{C}$ NMR (DMSO- $\left.d_{6}\right): 163.1(\mathrm{C}=\mathrm{O}), 153.1(\mathrm{C}=\mathrm{N}), 150.8(\mathrm{C}-4)$, 136.3 (ArH), 132.3 (C-2), 130.0 (C-1), 129.5 (C-6), 128.7 (ArH), 128.4 (ArH), 127.6 (ArH), 116.2 (C-5), $110.3(\mathrm{C}-3), 76.2\left(\mathrm{CH}_{2}\right)$, $38.2\left(\mathrm{CH}_{2}\right), 37.3\left(\mathrm{CH}_{2}\right), 28.8\left(\mathrm{CH}_{2}\right)$, HRMS (FAB): $m / z$ calcd for $\mathrm{C}_{36} \mathrm{H}_{36} \mathrm{Br}_{2} \mathrm{~N}_{4} \mathrm{O}_{6} \mathrm{~S}_{2}[\mathrm{M}+\mathrm{H}]^{+}$843.0521, found 843.0517.

(2) $N, N^{\prime}$-Bis(3-(4-hydroxyphenyl)-2-oximinopropionyl) cystamine (5)

Yield: 39\%, mp: $160-162^{\circ} \mathrm{C},{ }^{1} \mathrm{H}$ NMR (DMSO- $\left.d_{6}\right): 11.73(2 \mathrm{H}$, s, $2 \cdot \mathrm{OH}), 9.15(2 \mathrm{H}, \mathrm{s}, 2 \cdot \mathrm{OH}), 8.02(2 \mathrm{H}, \mathrm{t}, J=5.6 \mathrm{~Hz}, 2 \cdot \mathrm{NH})$, $7.00\left(4 \mathrm{H}, \mathrm{d}, J=8.4 \mathrm{~Hz}, \mathrm{H}-2,2^{\prime}, 6,6^{\prime}\right), 6.62(4 \mathrm{H}, \mathrm{d}, J=8.4 \mathrm{~Hz}$, $\left.\mathrm{H}-3,3^{\prime}, 5,5^{\prime}\right), 3.68\left(4 \mathrm{H}, \mathrm{s}, 2 \cdot \mathrm{CH}_{2}\right), 3.44(4 \mathrm{H}, \mathrm{q}, J=7.2 \mathrm{~Hz}$, $\left.2 \cdot \mathrm{CH}_{2}\right), 2.76$ (4H, t, $\left.J=7.6 \mathrm{~Hz}, 2 \cdot \mathrm{CH}_{2}\right),{ }^{13} \mathrm{C}$ NMR (DMSO- $d_{6}$ ): $163.1(\mathrm{C}=\mathrm{O}), 155.9(\mathrm{C}=\mathrm{N}), 151.6(\mathrm{C}-4), 131.3(\mathrm{C}-2,6), 127.8$ (C-1), 117.1 (C-3,5), 40.1 ( $\left.\mathrm{CH}_{2}\right)$, 38.1 $\left(\mathrm{CH}_{2}\right), 29.8\left(\mathrm{CH}_{2}\right)$, HRMS (FAB): $m / z$ calcd for $\mathrm{C}_{22} \mathrm{H}_{26} \mathrm{~N}_{4} \mathrm{O}_{6} \mathrm{~S}_{2}[\mathrm{M}+\mathrm{H}]^{+} 507.1372$, found 507.1370 .

(3) $N, N^{\prime}$-Bis(3-(4-hydroxyphenyl)-2-benzyloximinopropionyl) cystamine (6)

Yield: $38 \%,{ }^{1} \mathrm{H}$ NMR (DMSO- $\left.d_{6}\right)$ : 7.29-7.38 $(10 \mathrm{H}, \mathrm{m}, \mathrm{ArH})$, $7.14\left(4 \mathrm{H}, \mathrm{d}, J=10.0 \mathrm{~Hz}, \mathrm{H}-2,2^{\prime}, 6,6^{\prime}\right), 6.69(4 \mathrm{H}, \mathrm{d}, J=9.6 \mathrm{~Hz}$, H-3,3',5,5'), $5.19\left(4 \mathrm{H}, \mathrm{s}, 2 \cdot \mathrm{CH}_{2}\right), 3.89\left(4 \mathrm{H}, \mathrm{s}, 2 \cdot \mathrm{CH}_{2}\right), 3.51(4 \mathrm{H}$, q, $\left.J=7.6 \mathrm{~Hz}, 2 \cdot \mathrm{CH}_{2}\right), 2.69\left(4 \mathrm{H}, \mathrm{t}, J=8.8 \mathrm{~Hz}, 2 \cdot \mathrm{CH}_{2}\right),{ }^{13} \mathrm{C} \mathrm{NMR}$ (DMSO- $\left.d_{6}\right): 165.2(\mathrm{C}=\mathrm{O}), 155.1(\mathrm{C}=\mathrm{N}), 154.5(\mathrm{C}-4), 135.2$ (ArH), 132.2 (C-2,6), 128.2 (C-1), 128.7 (ArH), 128.5 (ArH), 127.8 (ArH), 116.3 (C-3,5), 76.2 ( $\left.\mathrm{CH}_{2}\right), 39.1\left(\mathrm{CH}_{2}\right), 38.2\left(\mathrm{CH}_{2}\right)$, $29.9\left(\mathrm{CH}_{2}\right)$, HRMS (FAB): $m / z$ calcd for $\mathrm{C}_{36} \mathrm{H}_{38} \mathrm{~N}_{4} \mathrm{O}_{6} \mathrm{~S}_{2}[\mathrm{M}+\mathrm{H}]^{+}$ 687.2311, found 687.2307.

\section{(4) N,N'-Bis(3,4-Difluorocinnamoyl) cystamine (8)}

Yield: $73 \%$, mp: $160-162^{\circ} \mathrm{C},{ }^{1} \mathrm{H}$ NMR (DMSO- $\left.d_{6}\right): 8.33(2 \mathrm{H}$, $\mathrm{t}, J=5.6 \mathrm{~Hz}, \mathrm{NH}), 7.63\left(2 \mathrm{H}, \mathrm{dd}, J=5.6,8.4 \mathrm{~Hz}, \mathrm{H}-6,6^{\prime}\right), 7.36-7.48$ (6H, m, 2 · CH, H-2,2',5,5'), $6.62(2 \mathrm{H}, \mathrm{d}, J=16.0 \mathrm{~Hz}, 2 \cdot \mathrm{CHCO})$, $3.48\left(4 \mathrm{H}, \mathrm{q}, J=6.4 \mathrm{~Hz}, 2 \cdot \mathrm{CH}_{2}\right), 2.86\left(4 \mathrm{H}, \mathrm{t}, J=6.8 \mathrm{~Hz}, 2 \cdot \mathrm{CH}_{2}\right)$, ${ }^{13} \mathrm{C}$ NMR (DMSO- $d_{6}$ ): 164.6 (CO), 149.9 (C-3, dd, $J=12.0,246.2$ $\mathrm{Hz}), 149.7$ (C-4, dd, $J=12.0,239.0 \mathrm{~Hz}), 136.7$ ( = CH), 132.7 (C-1, t, $J=4.2 \mathrm{~Hz}$ ), 124.6 (C-6, dd, $J=2.0,17.6 \mathrm{~Hz}), 123.2$ (= $\underline{\mathrm{CHCO}}$ ), 117.8 (C-5, dd, $J=4.0,17.8 \mathrm{~Hz}), 116.1(\mathrm{C}-2, J=17.9 \mathrm{~Hz}), 38.0$ $\left(\mathrm{CH}_{2}\right), 37.1\left(\mathrm{CH}_{2}\right)$, HRMS (FAB): $m / z$ calcd for $\mathrm{C}_{22} \mathrm{H}_{20} \mathrm{BrF}_{4} \mathrm{~N}_{2}$ 
$\mathrm{O}_{2} \mathrm{~S}_{2}[\mathrm{M}+\mathrm{H}]^{+} 485.0981$, found 485.0978.

(5) $N, N^{\prime}$-Bis(cinnamoyl)cystamine (9)

Yield: $78 \%$, mp: $121-123^{\circ} \mathrm{C},{ }^{1} \mathrm{H}$ NMR (DMSO- $\left.d_{6}\right): 8.35(2 \mathrm{H}$, t, $J=5.6 \mathrm{~Hz}, 2 \cdot \mathrm{NH}), 7.55$ (4H, d, $\left.J=6.8, \mathrm{~Hz}, \mathrm{H}-2,2^{\prime}, 6,6^{\prime}\right), 7.44$

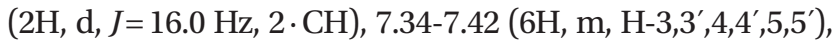
$6.64(2 \mathrm{H}, \mathrm{d}, J=16.0 \mathrm{~Hz}, 2 \cdot \mathrm{CHCO}), 3.50(4 \mathrm{H}, \mathrm{q}, J=6.4 \mathrm{~Hz}, 2$. $\mathrm{CH}_{2}$ ), $2.88\left(4 \mathrm{H}, \mathrm{t}, J=6.4 \mathrm{~Hz}, 2 \cdot \mathrm{CH}_{2}\right),{ }^{13} \mathrm{C}$ NMR (DMSO- $d_{6}$ ): 165.8 (CO), 139.6 (= CH), 135.5 (C-1), 130.1 (C-4), 129.6 (C-2,6), 128.2 (C-3,5), 122.6 (= CHCO), $38.8\left(\mathrm{CH}_{2}\right), 38.0\left(\mathrm{CH}_{2}\right)$, HRMS (FAB): $m / z$ calcd for $\mathrm{C}_{22} \mathrm{H}_{24} \mathrm{~N}_{2} \mathrm{O}_{2} \mathrm{~S}_{2}[\mathrm{M}+\mathrm{H}]^{+} 413.1357$, found 413.1353.

(6) $N, N^{\prime}$-Bis(2-(2-chlorophenyl)acetyl)cystamine (10)

Yield: $74 \%,{ }^{1} \mathrm{H}$ NMR (DMSO- $\left.d_{6}\right): 8.24(2 \mathrm{H}, \mathrm{t}, J=5.2 \mathrm{~Hz}, 2 \cdot \mathrm{NH}$ ), 7.40 ( $\left.2 \mathrm{H}, \mathrm{dd}, J=2.4,6.6 \mathrm{~Hz}, \mathrm{H}-3,3^{\prime}\right), 7.34(2 \mathrm{H}, \mathrm{dd}, J=2.0,6.4 \mathrm{~Hz}$, H-6,6'), 7.22-7.32 (4H, m, H-4,4',5,5'), 3.57 (4H, s, $\left.2 \cdot \mathrm{CH}_{2}\right), 3.56$ (4H, q, $\left.J=6.0 \mathrm{~Hz}, 2 \cdot \mathrm{CH}_{2}\right), 2.79\left(4 \mathrm{H}, \mathrm{t}, J=6.8 \mathrm{~Hz}, 2 \cdot \mathrm{CH}_{2}\right) ;{ }^{13} \mathrm{C}$ NMR (DMSO- $d_{6}$ ): $170.5(\mathrm{C}=$ O), $134.3(\mathrm{C}-1), 134.2(\mathrm{C}-6), 132.6$ (C-2), 129.7 (C-3), 129.3 (C-4), 127.8 (C-5), $40.5\left(\mathrm{CH}_{2}\right), 38.6$ $\left(\mathrm{CH}_{2}\right), 37.6\left(\mathrm{CH}_{2}\right)$, HRMS (FAB): $m / z$ calcd for $\mathrm{C}_{20} \mathrm{H}_{22} \mathrm{Cl}_{2} \mathrm{~N}_{2} \mathrm{O}_{2}$ $\mathrm{S}_{2}[\mathrm{M}+\mathrm{H}]^{+}$457.0578, found 457.0575.

(7) $N, N^{\prime}$-Bis(2-(3-methoxyphenyl)acetyl)cystamine (11)

Yield: $81 \%$, mp: $108-110^{\circ} \mathrm{C},{ }^{1} \mathrm{H}$ NMR (DMSO- $\left.d_{6}\right): 8.22(2 \mathrm{H}$, t, $J=5.2 \mathrm{~Hz}, 2 \cdot \mathrm{NH}), 7.19\left(2 \mathrm{H}, \mathrm{t}, J=7.6 \mathrm{~Hz}, \mathrm{H}-5,5^{\prime}\right), 6.76-6.85$ (6H, m, H-2,2',4,4',6,6'), 7.22-7.32 (4H, m, H-4,4',5,5'), 3.72 $\left(6 \mathrm{H}, \mathrm{s}, 2 \cdot \mathrm{CH}_{3}\right), 3.38\left(4 \mathrm{H}, \mathrm{s}, 2 \cdot \mathrm{CH}_{2}\right), 3.32(4 \mathrm{H}, \mathrm{q}, J=6.4 \mathrm{~Hz}$, $2 \cdot \mathrm{CH}_{2}$ ), 2.76 (4H, t, $\left.J=6.4 \mathrm{~Hz}, 2 \cdot \mathrm{CH}_{2}\right),{ }^{13} \mathrm{C}$ NMR (DMSO- $d_{6}$ ): 171.4 (C= O), 159.8 (C-3), 138.2 (C-1), 130.0 (C-5), 121.9 (C-6), $115.3(\mathrm{C}-2), 112.5(\mathrm{C}-4), 55.6\left(\mathrm{OCH}_{3}\right), 43.0\left(\mathrm{CH}_{2}\right), 38.6\left(\mathrm{CH}_{2}\right)$, $37.7\left(\mathrm{CH}_{2}\right)$, HRMS (FAB): $m / z$ calcd for $\mathrm{C}_{22} \mathrm{H}_{26} \mathrm{~N}_{2} \mathrm{O}_{4} \mathrm{~S}_{2}[\mathrm{M}+\mathrm{H}]^{+}$ 448.1490 , found 448.1487 .

\section{(8) $N, N^{\prime}$-Dibenzoylcystamine (12)}

Yield: $80 \%$, mp: $132-134^{\circ} \mathrm{C}$ (lit. $130-132^{\circ} \mathrm{C}$ [25]), ${ }^{1} \mathrm{H}$ NMR (DMSO- $d_{6}$ ): 8.65 (2H, s, 2 - NH), $7.83\left(4 \mathrm{H}, \mathrm{d}, J=7.6 \mathrm{~Hz}, \mathrm{H}-2,2^{\prime}\right.$, 6, $\left.6^{\prime}\right), 7.40-7.60$ (6H, m, H-3,3', $\left.4,4^{\prime}, 5,5^{\prime}\right), 3.56$ ( $4 \mathrm{H}, \mathrm{q}, J=6.0 \mathrm{~Hz}$, $2 \cdot \mathrm{CH}_{2}$ ), $2.93\left(4 \mathrm{H}, \mathrm{t}, J=6.4 \mathrm{~Hz}, 2 \cdot \mathrm{CH}_{2}\right),{ }^{13} \mathrm{C}$ NMR (DMSO- $d_{6}$ ): 168.0 (C=O), 134.5 (C-1), 132.3 (C-4), 129.2 (C-3,5), 127.7 (C-2,6), $38.7\left(\mathrm{CH}_{2}\right), 37.7\left(\mathrm{CH}_{2}\right)$, HRMS (FAB): $m / z$ calcd for $\mathrm{C}_{18} \mathrm{H}_{20} \mathrm{~N}_{2} \mathrm{O}_{2} \mathrm{~S}_{2}[\mathrm{M}+\mathrm{H}]^{+}$361.1044, found 361.1040.

\section{(9) N-Cinnamoylcysteamine (13)}

To a solution of $N, N^{\prime}$-bis(cinnamoyl)cystamine $(9,412 \mathrm{mg}$, $1 \mathrm{mmol})$ in methanol (30 $\mathrm{mL}$ ) and dimethylformamide (5 mL),
$1 \mathrm{M} \mathrm{KOH}(0.1 \mathrm{~mL})$ and dithiothreitol (462 mg, $3 \mathrm{mmol}$ ) were added at room temperature. The reaction mixture was quenched with $0.5 \mathrm{M} \mathrm{HCl}$ at $0^{\circ} \mathrm{C}$ after 1 hour and extracted with dichloromethane $(3.30 \mathrm{~mL})$. The combined extracts were washed with water and brine, and were dried over anhydrous $\mathrm{MgSO}_{4}$, and then filtered. The filtrate was evaporated to give crude oily compound. The desired compound 13 was obtained as a colorless oil by short column chromatography (ethyl acetate: $n$-hexane $=1: 1$ ). Yield: $88 \%,{ }^{1} \mathrm{H}$ NMR (DMSO- $\left.d_{6}\right): 8.33(1 \mathrm{H}, \mathrm{t}, J=5.6 \mathrm{~Hz}, \mathrm{NH}), 7.53(1 \mathrm{H}, \mathrm{d}, J=6.8 \mathrm{~Hz}$, $\mathrm{H}-2,6), 7.42(1 \mathrm{H}, \mathrm{d}, J=16.0 \mathrm{~Hz},=\mathrm{CH}), 7.35-7.43(3 \mathrm{H}, \mathrm{m}, \mathrm{H}-3$, 4,5), $6.63(1 \mathrm{H}, \mathrm{d}, J=16.0 \mathrm{~Hz},=\mathrm{CHCO}), 3.48(2 \mathrm{H}, \mathrm{q}, J=6.8 \mathrm{~Hz}$, $\left.\mathrm{CH}_{2}\right), 2.79\left(2 \mathrm{H}, \mathrm{q}, J=6.4 \mathrm{~Hz}, \mathrm{CH}_{2}\right), 2.43(1 \mathrm{H}, \mathrm{t}, J=6.8 \mathrm{~Hz}, \mathrm{SH})$, ${ }^{13} \mathrm{C}$ NMR (DMSO- $d_{6}$ ): $164.9(\mathrm{C}=\mathrm{O}), 139.2(=\mathrm{CH}), 134.8(\mathrm{C}-1)$, 129.2 (C-2,6), 127.9 (C-3,5), 122.0 (= $\underline{\mathrm{CHCO}}$ ), $38.5\left(\mathrm{CH}_{2}\right), 37.5$ $\left(\mathrm{CH}_{2}\right)$, HRMS (FAB): $m / z$ calcd for $\mathrm{C}_{11} \mathrm{H}_{13} \mathrm{NOS}[\mathrm{M}+\mathrm{H}]^{+} 208$. 0796, found 208.0792.

\section{Cell Culture}

A549 (human lung cancer) and U373MG (human glioblastoma) cell lines were purchased from the Korean Cell Line Bank. Cells were cultured and maintained in RPMI media (Welgene, Daegu, Korea), supplemented with $10 \%$ fetal bovine serum and $12.5 \mu \mathrm{g} \cdot \mathrm{mL}^{-1}$ of gentamicin. All compounds were dissolved in DMSO as concentrated stock solutions at $20 \mathrm{mM}$ concentration, corresponding to concentrations of $2.66,3.37,2.02,1.94,1.65,1.83,1.79,1.44$, and $0.83 \mathrm{mg} / 0.2 \mathrm{~mL}$ for PsA, 4, 5, and 8-13, respectively. They were stored at $-20^{\circ} \mathrm{C}$, and diluted at the time of use.

\section{Clonogenic assay}

Cell survival after treatment with PsA-modified derivatives and/or irradiation was measured using clonogenic assay as previously reported $[21,22]$. Briefly, cells were trypsinized, and appropriate number of cells were seeded into 6 well plates and incubated for 24 hours. Then, cells were treated with nine synthesized compounds or DMSO (for the drugfree groups) at specified concentrations for additional 24 hours. After specified drug treatment, the media was replaced with fresh drug-free medium. Then, to determine the $50 \%$ inhibitory concentrations $\left(\mathrm{IC}_{50}\right.$ ) of each compound, cells were incubated for another 12 days to allow for colony formation. To screen for the radiosensitizing properties of each compound, cells were irradiated using 6 MVX-ray from a linear accelerator (Clinac 6EX or Clinac 21EX, Varian Medical systems, Palo Alto, USA) at a dose of 0, 2, 4, 6, and 8 Gy 
immediately after the media was changed (post-seeding 48 hours), and then also incubated for 12 additional days. Then, colonies were fixed with methanol, stained with $0.5 \%$ crystal violet, and counted. Only colonies containing 50 cells or more were counted.

\section{DNMT1 inhibition assay}

Assaying DNMT1 inhibitory activity of compounds was

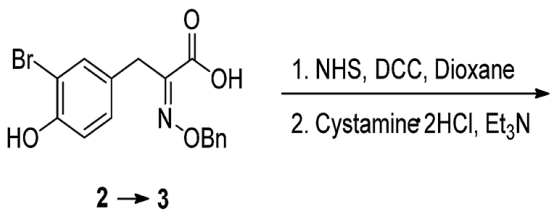

$\mathrm{KBr}, \mathrm{KBrO}_{3}$ $5 \% \mathrm{HCl}, \mathrm{RT}$<smiles>[R20]N=C(Cc1ccc(O)c(Br)c1)C(=O)NCCSSCCNC(=O)/C(Cc1ccc(O)c(Br)c1)=N/O[R]</smiles>

$4: \mathrm{R}=\mathrm{OBn}$

Psammaplin A ; $\mathrm{R}=\mathrm{H}$

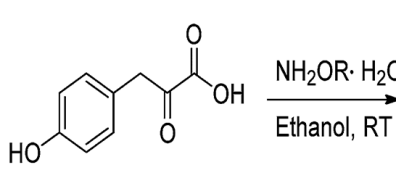<smiles>[R]O/N=C(/Cc1ccc(O)cc1)C(=O)O</smiles><smiles>[R20]N=C(Cc1ccc(O)cc1)C(=O)NCCSSCCNC(=O)C(Cc1ccc(O)cc1)=NO[R]</smiles><smiles>O=Cc1ccc(F)c(F)c1</smiles>

Malonic acid Piperidine, Pyridine<smiles>O=C(O)/C=C/c1ccc(F)c(F)c1</smiles>
$\underset{\text { 2. Cystamine } 2 \mathrm{HCl}, \mathrm{Et}_{3} \mathrm{~N}}{\stackrel{\mathrm{NHS}, \mathrm{DCC}, \text { Dioxane, } \mathrm{RT}}{\longrightarrow}}$ $\mathrm{RT}$<smiles>O=C(/C=C/c1ccc(F)c(F)c1)NCCSSCCNC(=O)/C=C/c1ccc(F)c(F)c1</smiles>

7<smiles>O=C(O)/C=C/c1ccccc1</smiles>

\section{NHS, DCC, Dioxane, RT 2. Cystamine $2 \mathrm{HCl}, \mathrm{Et}_{3} \mathrm{~N}$} RT<smiles>O=C(/C=C/c1ccccc1)NCCSSCCNC(=O)/C=C/c1ccccc1</smiles>

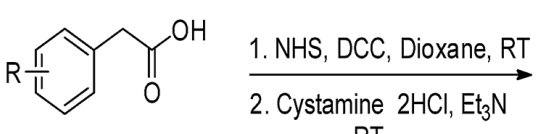
RT<smiles>[R][R]1cccc(CC(=O)NCCSSCCNC(=O)Cc2cc[R]cc2)c1</smiles>

$10: R=2-C$

$11: \mathrm{R}=3-\mathrm{OCH}_{3}$<smiles>CCN(CC)CC(=O)O[Mg]</smiles>

RT<smiles>O=C(NCCSSCCNC(=O)c1ccccc1)c1ccccc1</smiles>

12
$9 \underset{1 \mathrm{M} \mathrm{KOH} \text {, Ethanol }}{\stackrel{\text { Cleland's reagent }}{\longrightarrow}}$ RT<smiles>O=C(/C=C/c1ccccc1)NCCS</smiles>

Scheme 1. Synthesis of homodimeric disulfides (4-6 and 8-12) and a monomer (13). 
done using the colorimetric EpiQuik DNMT activity/inhibition assay Ultra kit (Epigentek, Farmingdale, USA) according to the manufacturer's instructions. Briefly, cells were treated with compounds 5 and 10 at a concentration of $100 \mu \mathrm{M}$ for 24 hours, and DNA was extracted from cells. Then, the colorimetric assay for methylated DNA was performed in 96-well plates. A microplate reader with a $450-\mathrm{nm}$ filter read each plate. The DNMT1 activity was proportional to sample optical density (OD). DNMT1 inhibition was calculated as follows: DNMT1 inhibition (\%) = [1-(inhibitor sample ODblank OD)] $100 /$ (no inhibitor sample OD-blank OD). All data were obtained from triplicate experiments.

\section{Statistical analysis}

Cell survival data were presented as means of surviving fractions from triplicate experiments. Kaleidagraph version 3.51 (Synergy Software, Reading, USA) was used to fit surviving fractions of irradiated cells to a linear quadratic model of radiation cell killing. Parameters of fitted linear quadratic models were used to calculate radiation doses to obtain specified surviving fractions. Sensitizer enhancement ratio (SER) at surviving fraction of 0.2 was calculated as previously reported [21, 22]. To determine whether the required radiation dose for surviving fraction of 0.2 was significantly lower in the group treated with both PsA-modified derivative and radiation compared to the group which received radiation only from triplicate experiments of a PsA derivative, the ratio paired $t$ test was performed. The level of statistical significance was set at $p<0.05$. Statistical Package for the Social Sciences version 22.0 (IBM SPSS, Armonk, USA) was used for statistical testing.

\section{Results and Discussion}

\section{Synthesis}

Using PsA as a lead compound, nine PsA-modified compounds were synthesized. They are eight homodimers (4-6 and 8-12) and one monomer (13). As shown in scheme 1, 4-hydroxyphenylpyruvic acid was reacted with hydroxylamine and $O$-benzylhydroxylamine to afford oxime 1 and $O$ benzyloxime 2 , respectively. Compound 2 was brominated to form bromotyrosine analog 3 by $\mathrm{KBr}$ and $\mathrm{KBrO}_{3}$ in an acidic condition [26]. Compound 3 was reacted with cystamine dihydrochloride and triethylamine to synthesize bromotyrosine-derived disulfide analog 4 by $N$-hydroxysuccinimide (NHS) and dicyclohexylcarbodiimide (DCC) coupling
Table 1. Cytotoxicity of PsA and Its Derivatives

\begin{tabular}{ccc}
\hline \multirow{2}{*}{ Compound } & \multicolumn{2}{c}{$\mathrm{IC}_{50}(\mu \mathrm{M}$, mean $\pm \mathrm{SD})$} \\
\cline { 2 - 3 } & $\mathrm{A} 549$ & $\mathrm{U} 373 \mathrm{MG}$ \\
\hline PsA & $4.01 \pm 1.87$ & $5.39 \pm 0.71$ \\
4 & $45.40 \pm 3.46$ & $38.68 \pm 0.00$ \\
5 & $19.43 \pm 16.12$ & $20.84 \pm 11.25$ \\
6 & Not reached & Not reached \\
8 & $41.83 \pm 24.18$ & $23.04 \pm 2.22$ \\
9 & $16.14 \pm 6.52$ & $14.65 \pm 4.55$ \\
10 & $150.10 \pm 22.46$ & $50.15 \pm 9.21$ \\
11 & $22.55 \pm 7.70$ & $20.39 \pm 7.46$ \\
12 & $66.31 \pm 38.41$ & $13.25 \pm 7.09$ \\
13 & $97.55 \pm 21.48$ & $13.76 \pm 2.33$ \\
\hline
\end{tabular}

SD, standard deviation; PSA, psammaplin A; $\mathrm{C}_{50}, 50 \%$ inhibitory concentration.

reaction. The synthesis of homodimeric tyrosine-derived disulfide analog 5 and 6, which have no 3-bromo group in their structures, were achieved by amide coupling of compound 1 and 2 with cystamine dihydrochloride using NHS and DCC.

Apart from tyrosine-derived disulfide analogs, 3,4-difluorocinnamic acid (7), cinnamic acid, 2-chlorophenylacetic acid, 3-methoxyphenylacetic acid, and benzoic acid were coupled with cystamine dihydrochloride to afford four homodimeric disulfide analogs, 8-12 in moderate yields, respectively. Compound 7 was obtained from 3,4-difluorobenzaldehyde and malonic acid in the presence of piperidine and pyridine. $N, N^{\prime}$-Bis(cinnamoyl)cystamine (9) was reduced by dithiothreitol (Cleland's reagent) to afford $N$-cinnamoylcysteamine (13) as monomer.

\section{Inhibition of cell survival}

Clonogenic cell survivals for PsA and the nine synthesized compounds (4-6 and 8-13) were measured, and all compounds reduced the viability of cells in a dose-dependent manner. The $\mathrm{IC}_{50}$ values of compounds are shown in Table 1.

\section{Screening of the compounds as radiosensitizers}

Clonogenic cell survival was measured after combination of X-ray RT and treatment of seven compounds (4, 5, 8-11, and 13) at measured respective $\mathrm{IC}_{50} \mathrm{~S}$ for 24 hours prior to $\mathrm{X}$ ray RT. However, since compound 5, 8, 9, and 11 were not shown to radiosensitize A549 and U373MG cells at the concentration of around each $\mathrm{IC}_{50}$ value, concentrations of the compounds were subsequently increased (concentration displayed on Figure 1). Radiation survival curves were compared between cells treated with synthesized compounds and those treated with DMSO as the control (Figure 1). The 

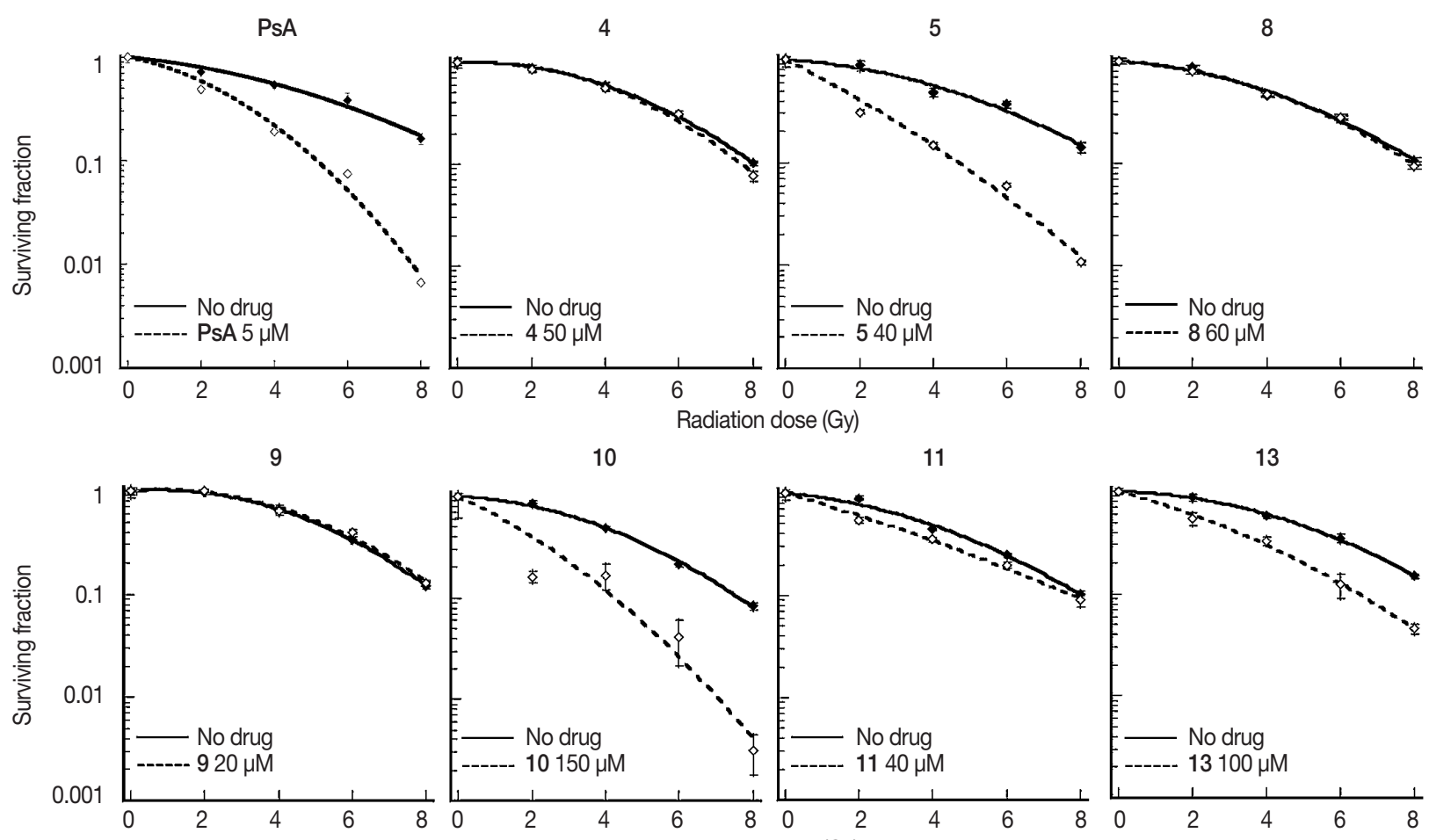

10

11

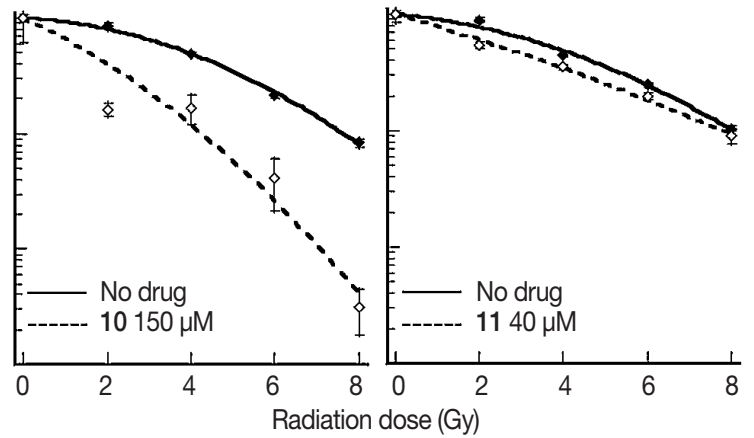

13

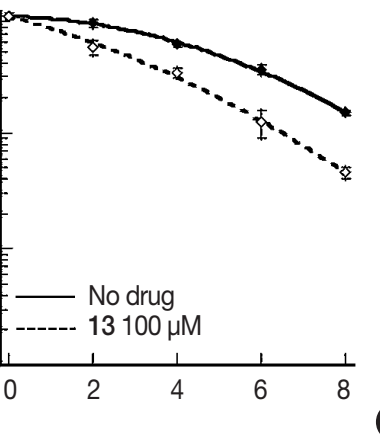

PsA

4

5

8
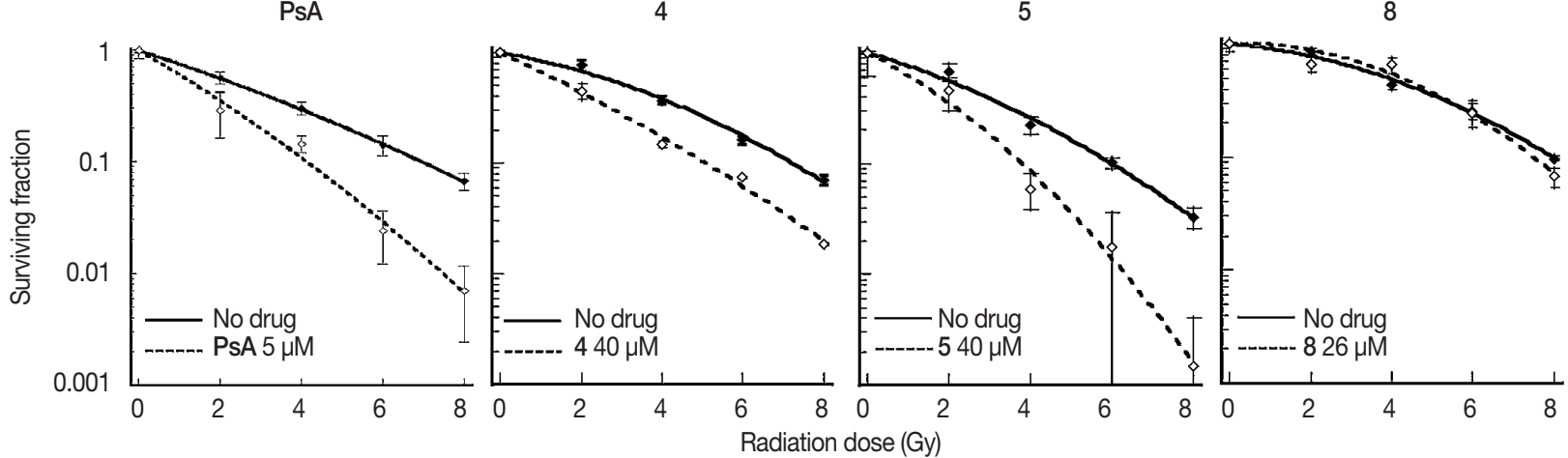

9

10

11

13
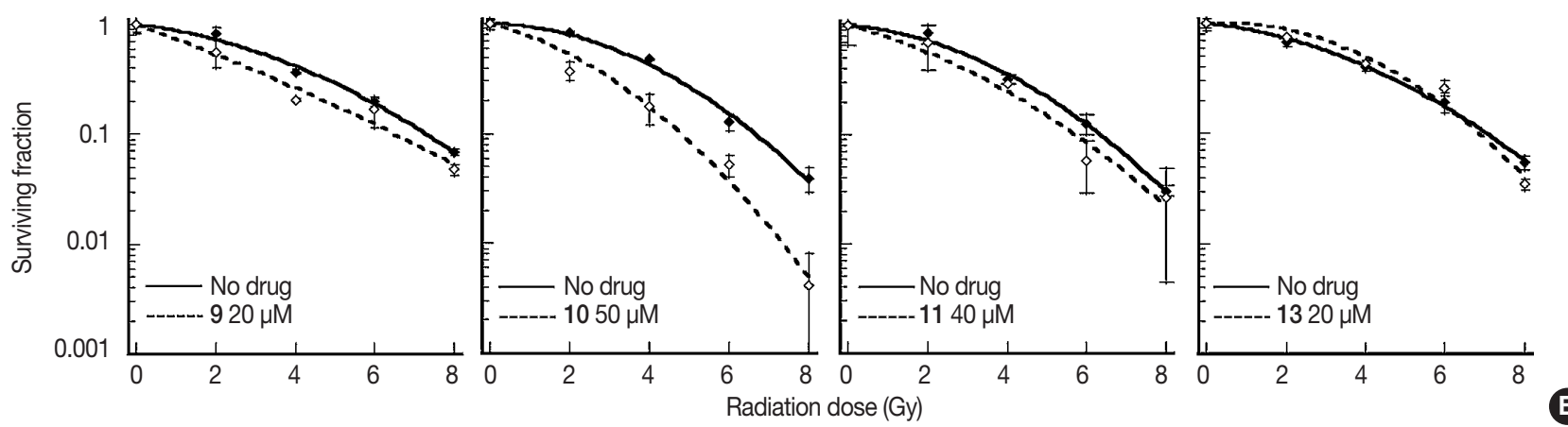

B

Fig. 1. Clonogenic cell survival in (A) A549 and (B) U373MG cells after combination of irradiation and treatment of synthesized compounds. Cells were treated with compounds for 24 hours before irradiation to graded doses of $\mathrm{X}$-ray. Surviving fractions are shown mean $\pm \mathrm{SD}$ from triplicate experiments. PsA, psammaplin A. 

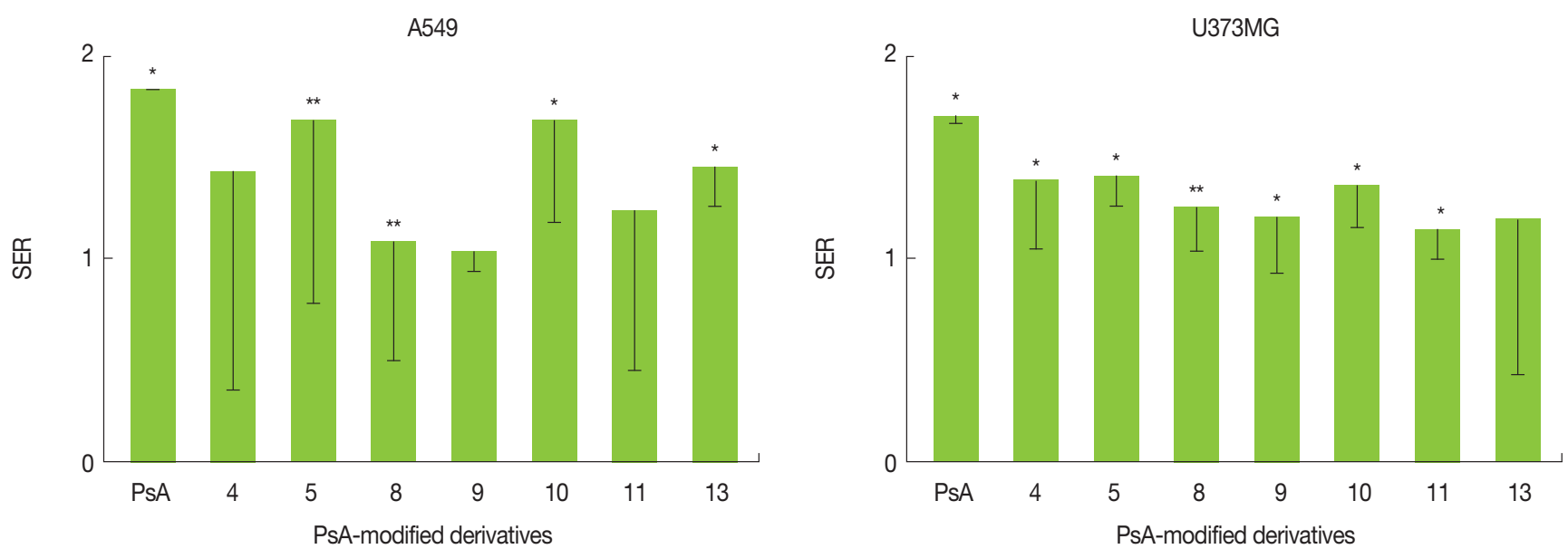

Fig. 2. Sensitizer enhancement ratio (SER) of synthetized compounds. SER is a ratio of radiation doses necessary in the absence of each compound compared to in the presence of each compound to produce a surviving fraction of 0.2. SERs are shown as mean with lower limits of the $95 \%$ confidence intervals $(n=3) .{ }^{*} p<0.05,{ }^{\star *} p<0.1$ by ratio paired $t$ test. PsA, psammaplin A.

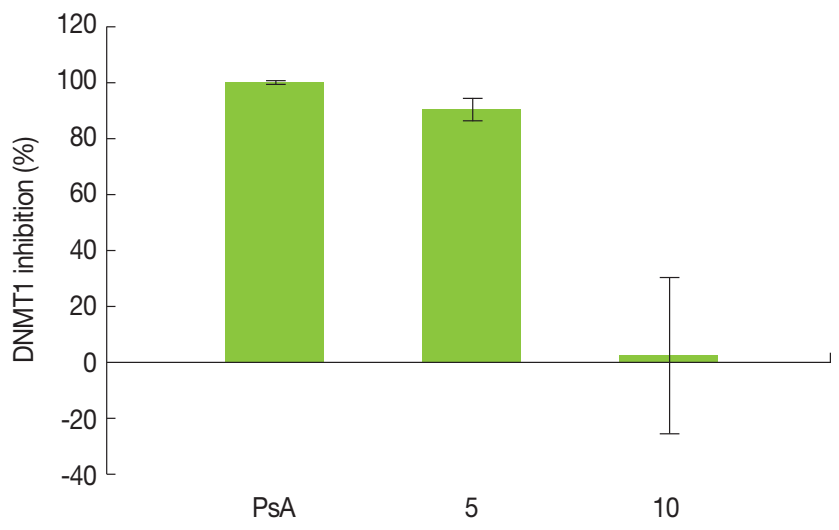

Fig. 3. Inhibition of DNMT1 activity by psammaplin A (PsA), 5 and 10 when treated at a concentration of $100 \mu \mathrm{M}$ for 24 hours. DNMT1 activity was assayed using a DNMT activity assay kit. All data are shown as mean $\pm S D(n=3)$.

surviving fractions of each compound were measured in a range $0-8 \mathrm{~Gy}$ and shown as the mean \pm SD from triplicate experiments.

The SERs are shown in Figure 2. Two compounds (10 and 13) significantly $(p<0.05)$, and two other compounds (5 and 8) marginally $(p<0.10)$ enhanced cell killing by RT in A549 cells. Five compounds (4, 5, and 9-11) induced significant $(p<0.05)$ radiosensitization whereas one compound (8) demonstrated a marginal $(p<0.10)$ radiosensitization in U373MG cells. Compounds 5, 8, and 10 showed at least marginal radiosensitization in both cell lines. However, although statistically marginal, 8 minimally radiosensitized A549 cells with a mean SER value of 1.08 . Thus, only 5 and 10 were selected for DNMT1 inhibition assay.

\section{Inhibition of DNMT1 activity}

DNMT1 activity was significantly inhibited by PsA and compound 5 at $100 \mu \mathrm{M}$, with inhibition rates of $99.99 \%$ and $90.57 \%$, respectively. However, DNMT activity was not affected by treatment with 10 (Figure 3).

\section{Discussion}

The therapeutic ratio of RT can be improved by chemical agents that sensitize cancer cell to the cytotoxic effects of ionizing radiation. We have previously reported that PsA is a potent radiosensitizer in vitro with poor bioavailability in vivo [21, 22]. The present study aimed to synthesize novel compounds that retain radiosensitizing properties of PsA. Such strategy has successfully generated a novel histone deacetylase inhibitor with both in vivo radiosensitizing capacity and biostability $[27,28]$.

Lee et al. [29] reported that 4-hydroxy modified (4-benzyloxy, 4-tert-butyl, 4-ethoxy-) derivatives of PsA which have no 3-bromo group in their structure were synthesized and demonstrated improved pharmacokinetic properties. In our study, since the oxime is known as an unstable group, one chemical approach to improve the bioavailability was to mask it by protection. We protected the oxime unit of PsA with benzyl group to afford $O$-benzyl oxime analog 4 . For further study on how the bromo group of PsA influenced radiosensitivity, oxime analog 5 and $O$-benzyl oxime analog 6 , which have no 3-bromo groups were synthesized.

There is a limitation in commercial availability of tyrosine and phenylpyruvic acid to synthesize the derivatives of PsA 
with diverse aromatic substitution patterns. Structurally, the PsA derivatives are composed of a cap structure and a linker which can prepare the homodimer, monomer, and heterodimer. We adopted a commercially available carboxylic acid containing aromatic ring instead of tyrosine moiety as a cap substructure, which would allow better physicochemical properties. Fluoro group may increase a target compound's lipophilicity and is considered importantly when obtaining molecules that are designed to be active in vivo. Fluorine can also aid hydrophobic interactions between the drug and binding sites on receptors or enzymes [30]. The chlorine substituent is known as a more lipophilic group than a fluoro- or methoxy- substituent, so it was selected for improving the bioavailability. 3,4-Difluorocinnamic acid, cinnamic acid, 2-chlorophenylacetic acid, 3-methoxyphenylacetic acid and benzoic acid, instead of the tyrosine part (4-hydroxyphenylpyruvic acid), were used for the synthesis of five homodimeric disulfide derivatives (8-12).

As shown in Figures 2 and 3, compounds 5, 8, 10, and 13 radiosensitized the A549 cells, and 4, 5, and 9-11 radiosensitized the U373MG cells, in vitro. Especially, $N, N^{\prime}$-bis(3-(4hydroxyhenyl)-2-oximinopropionyl)cystamine (5) and $N, N^{\prime}$ bis(2-(2-chlorophenyl)acetyl)cystamine (10) enhanced the radiation lethality by around 1.5 times in both cell lines. Both 5 and 10 are of a homodimeric disulfide structure, and are potent radiosensitizers comparable to PsA (Figure 2). However, $O$-benzyl oxime analog 4 did not demonstrate a statistically significant radiosensitizing effect in A549 cells. The benzyl protection of oxime seemed to result in reduced radiosensitization. Future studies should be performed whether the radiosensitizing effects of the compounds are affected by drug concentration.

Along with histone deacetylation, DNA methylation is a major mechanism of epigenetic alteration. Thus, inhibitors of epigenetic modulators such as histone deacetylase and DNMT have been investigated for anti-tumor activity in clinical trials. Inhibition of DNMT as well as histone deacetylase has been reported to sensitize human cancer cells to radiation-induced cell killing [21, 22]. However, no epigenetic modulators are currently used in conjunction with RT in clinical settings partly due to biochemical instability.

We have previously reported that a possible underlying mechanism of radiosensitization by a known DNMTI, PsA, is via inhibiting the DNA damage repair process [21]. Since 5 and 10 are PsA-modified homodimeric disulfides, we evaluated the inhibition effect on DNMT1 activity in 2 compounds at a concentration of $100 \mu \mathrm{M}$. As a result, 5 showed DNMT1 inhibitory activity comparable to PsA. However, it is remarkable that, 10 did not show any inhibition of DNMT1 (Figure 3). It is unknown whether DNMT inhibitory activity is prerequisite of radiosensitization by PsA. Despite the absence of DNMT1 inhibitory activity, 10 was as a potent a radiosensitizer. Thus, our observations may suggest that compound 10 confers radiosensitizing properties regardless of DNMT1 inhibitory activity. However, since the concentration of compounds used for the DNMT inhibition assay was very high, these results of DNMT inhibition assay may not have a biological meaning. Furthermore, since no mechanistic investigation was performed, it is to be elucidated whether 10 inhibits different isotypes of DNMTs.

From this result, we fortunately found that the thiol monomers, the major fragment of disulfide derivatives, are similar with synthetic thiol compounds which are known as radiopropectors. Of these series, amifostine (WR-2721, Ethyol ${ }^{\mathbb{Q}}$, 2-(3-amniopropyl)ethylsulfanylphosphonic acid) is the only radioprotector that has been clinically approved by the FDA. An inactive prodrug, amifostine, is transformed to an active free thiol (WR-1065) by dephosphorylation by alkaline phosphatase in normal tissues. Generally, normal tissues are able to contain free thiol up to a concentration 100-fold higher than tumors and thus can the free thiols protect normal tissues selectively [31]. Like all other drugs, amifostine has side effects such as acute hypotension, severe nausea, vomiting and allergy when administered intravenously [32]. In the future, PsA-modified derivatives will be studied for the potential dual effects of radiosensitization and radioprotection.

\section{Conclusion}

Using PsA as a lead structure, we synthesized and identified nine compounds that showed potent in vitro radiosensitization in two human cancer cell lines, lung cancer and glioblastoma. The present study suggests that the synthesized compounds have a great potential to serve as structural backbones for development of novel radiosensitizers further clinical application. Future investigations are warranted to confirm the bioavailability, radiosensitizing properties in vivo, mechanism, and safety of the compounds.

\section{Acknowledgements}

Funding: This study was supported by the Korean govern- 
ment (MSIP) through the National Research Foundation of Korea grant 2013M2A2A7043683 (Il Han Kim), and the Seoul National University Hospital through SNUH CRI 0320170080 (Il Han Kim) and SNUH CRI 0320130400 (Hak Jae Kim). Authors thank Eun Jung Oh for her administrative and management support of the research project.

\section{References}

1. Begg AC, Stewart FA, Conchita V. Strategies to improve radiotherapy with targeted drugs. Nat. Rev. Cancer. 2011;11(4):239253.

2. Esteller M. Relevance of DNA methylation in the management of cancer. Lancet. Oncol. 2003;4(6):351-358.

3. Lyko F, Brown R. DNA Methyltransferase inhibitors and the development of epigenetic cancer therapies. J. Natl. Cancer. Inst. 2005;97(20):1498-1506.

4. Manoharan M, Ramachandran K, Soloway MS, Singal R. Epigenetic targets in the diagnosis and treatment of prostate cancer. Int. Braz. J. Urol. 2007;33(1):11-18.

5. Lin RK, Hsu HS, Chang JW, Chen CY, Chen JT, Wang YC. Alteration of DNA methyltransferases contributes to 5'CpG methylation and poor prognosis in lung cancer. Lung. Cancer. 2007; 55(2):205-213.

6. Eads CA, Danenberg KD, Kawakami K, Saltz LB, Danenberg PV, Laird PW. CpG island hypermethylation in human colorectal tumors is not associated with DNA methyltransferase overexpression. Cancer. Res. 1999;59(10):2302-2306.

7. Silverman LR, Demakos EP, Peterson BL. Randomized controlled trial of azacitidine in patients with the myelodysplastic syndrome: a study of the cancer and leukemia group B. J. Clin. Oncol. 2002;20(10):2429-2440.

8. Pechalrieu D, Etievant C, Arimondo PB. DNA methyltransferase inhibitors in cancer: From pharmacology to translational studies. Biochem. Pharmacol. 2017;129;1-13.

9. Kristensen LS, Nielsen HM, Hansen LL. Epigenetic and cancer treatment. Eur. J. Pharmacol. 2009;625(1-3):131-142.

10. Oki Y, Aoki E, Issa JP. Decitabine-bedside to bench. Crit. Rev. Oncol. 2007;61(2):140-152.

11. Dote H, Cerna D, Burgan WE, Carter DJ, Cerra MA, Hollingshead MG, Camphausen K, Tofilon PJ. Enhancement of in vitro and in vivo tumor cell radiosensitivity by the DNA methylation inhibitor zebularine. Clin. Cancer. Res. 2005;11(12):4571-4579.

12. Cho HJ, Kim SY, Kim KH, Kang WK, Kim JI, Oh ST, Kim JS, An $\mathrm{CH}$. The combination effect of sodium butyrate and 5-Aza-2'deoxycytidine on radiosensitivity in RKO colorectal cancer and MCF-7 breast cancer cell lines. World. J. Surg. Oncol. 2009;7:49.

13. Qiu H, Yashiro M, Shinto O, Matsuzaki T, Hirakawa K. DNA methyltransferase inhibitor 5-aza-CdR enhances the radiosen- sitivity of gastric cancer cells. Cancer. Sci. 2009;100(1):181-188.

14. De Schutter H, Kimpe M, Isebaert S, Nuyts S. A systematic assessment of radiation dose enhancement by 5 -aza-2'-deoxycytidine and histone deacetylase inhibitors in head-and-neck squamous cell carcinoma. Int. J. Radiat. Oncol. Biol. Phys. 2009;73(3):904912.

15. Lübbert M. DNA methylation inhibitors in the treatment of leukemias, myelodysplastic syndromes and hemoglobinopathies: clinical results and possible mechanisms of action. Curr. Top. Microbiol. Immunol. 2000;249:135-164.

16. Aparicio A, Weber JS. Review of the clinical experience with 5-azacytidine and 5-aza-2'-deoxycytidine in solid tumors. Curr. Opin. Investig. Drugs. 2002;3(4):627-633.

17. Arabshahi L, Schmitz FJ. Brominated tyrosine metabolites from an unidentified sponge. J. Org. Chem. 1987;52(16):3584-3586.

18. Piña IC, et al. Psammaplins from the sponge Pseudoceratina purpurea: inhibition of both histone deacetylase and DNA methyltransferase. J. Org. Chem. 2003;68:3866-3873.

19. Baud MG, et al. Defining the mechanism of action and enzymatic selectivity of psammaplin A against its epigenetic targets. J. Med. Chem. 2012;55(4):1731-1750.

20. Kim JH, Kim IH, Shin JH, Kim HJ, Kim IA. Sequence-dependent radiosensitization of histone deacetylase inhibitors trichostatin A and SK-7041. Cancer. Res. Treat. 2013;45(4):334-342.

21. Kim HJ, Kim JH, Chie EK, Park DY, Kim IA, Kim IH. DNMT (DNA methyltransferase) inhibitors radiosensitize human cancer cells by suppressing DNA repair activity. Radiat. Oncol. 2012;7:39.

22. Kim HJ, Kim TH, Seo WS, Yoo SD, Kim IH, Joo SH, Shin S, Park ES, Ma ES, Shin BS. Pharmacokinetics and tissue distribution of psammaplin A, a novel anticancer agent, in mice. Arch. Pharm. Res. 2012;35(10):1849-1854.

23. Löffelhardt $W$. The biosynthesis of phenylacetic acids in the blue-green alga Anacytis nidulans: Evidence for the involvement of a thylakoid-bound L-amino acid oxidase. Z. Naturforsch. 1977; 32(5-6):345-350.

24. Nicolaou KC, Hughes R, Pfefferkorn JA, Barluenga S, Roecker AJ. Combinational synthesis through disulfide exchange: Discovery of potent psammaplin A type antibacterial agent active against methicillin-resistant Staphylococcus aureus (MRSA). Chem. Eur. J. 2001;7(19):4280-4295.

25. Mautner HG, Chu SH, Gunther WHH. The aminolysis of thioacyl and selenoacyl analogs. J. Am. Chem. Soc. 1963;85(21):34583462.

26. Godert AM, Angelino N, Woloszynska-Read A, Morey SR, James SR, Karpf AR, Sufrin JR. An improved synthesis of psammaplin A. Bioorg. Med. Chem. Lett. 2006;16(12):3330-3333.

27. Kim DK, et al. Synthesis and biological evaluation of 3-(4-substituted-phenyl)-N-hydroxy-2-propenamides, a new class of histone deacetylase inhibitors. J. Med. Chem. 2003;46(26):5745-5751. 28. Chie EK, Shin JH, Kim IA, Kim IH. In vivo radiosensitization ef- 
fect of HDAC inhibitor, SK-7041 on RIF-1 cell line. J. Korean. Soc. Ther. Radiol. Oncol. 2010;28(4):219-223.

29. Lee JY, Lee MY, Ha MW, Won TH, Cho HJ, Shin J, Park HG, Kim DD. Determination and validation of psammaplin A and its derivatives in rat plasma by liquid chromatography-tandem mass spectrometry and its application in pharmacokinetic study. J. Chromatogr. B. 2015;1000:155-162.
30. Gillis EP, Eastman KJ, Hill MD, Donnelly DJ, Meanwell NA. Applications of fluorine in medicinal chemistry. J. Med. Chem. 2015; 58(21):8315-8359.

31. Wasserman TH, Brizel DM. The role of amifostine as a radioprotector. Oncology. 2001;15(10):1349-1360.

32. Hosseinimehr SJ. Trends in the development of radioprotective agents. Drug. Discovery. Today. 2007;12(19-20):794-805. 